\title{
Pneumonitis and extreme failure to thrive
}

\author{
KL Hon *, TF Leung, YS Yau
}

Hong Kong Med J 2014;20:556.e1-3

DOI: $10.12809 / \mathrm{hkmj} 134106$

Failure to thrive is an uncommon, challenging, 2 but important basket of differential diagnoses to manage in the city of Hong Kong. Diagnosis may 3. be non-organic or functional. During outbreaks of avian influenza in Mainland China and MERS/ SARI (Middle East respiratory syndrome/severe acute respiratory illness) coronavirus infection in the Middle East in early 2013, ${ }^{1}$ a 27 -month-old girl was brought by her parents to Hong Kong following a long period of hospitalisation and investigations in Mainland China for recurrent pneumonia, chronic diarrhoea, lymphadenopathy, oral candidiasis, and failure to thrive. Reportedly, no bacterial or viral pathogens had been found. Antenatal anti-human immunodeficiency virus (HIV) antibody testing was negative in the mother. There had been no adverse reaction to Bacille Calmette-Guérin and routine immunisations. The child had been exclusively breastfed and her growth and development were normal until 9 months of age. The child was admitted to the paediatric intensive care unit (ICU) of a Hong Kong hospital for management. She was noted to have profound failure to thrive with a body weight of only $5 \mathrm{~kg}$ (Fig 1). Chest X-ray showed diffuse pneumonitis (Fig 2). She required oxygen supplementation but mechanical ventilation was not needed.

Which of the following investigations will most likely give the underlying diagnosis?

1. White cell counts and differentials for congenital neutrophil abnormality

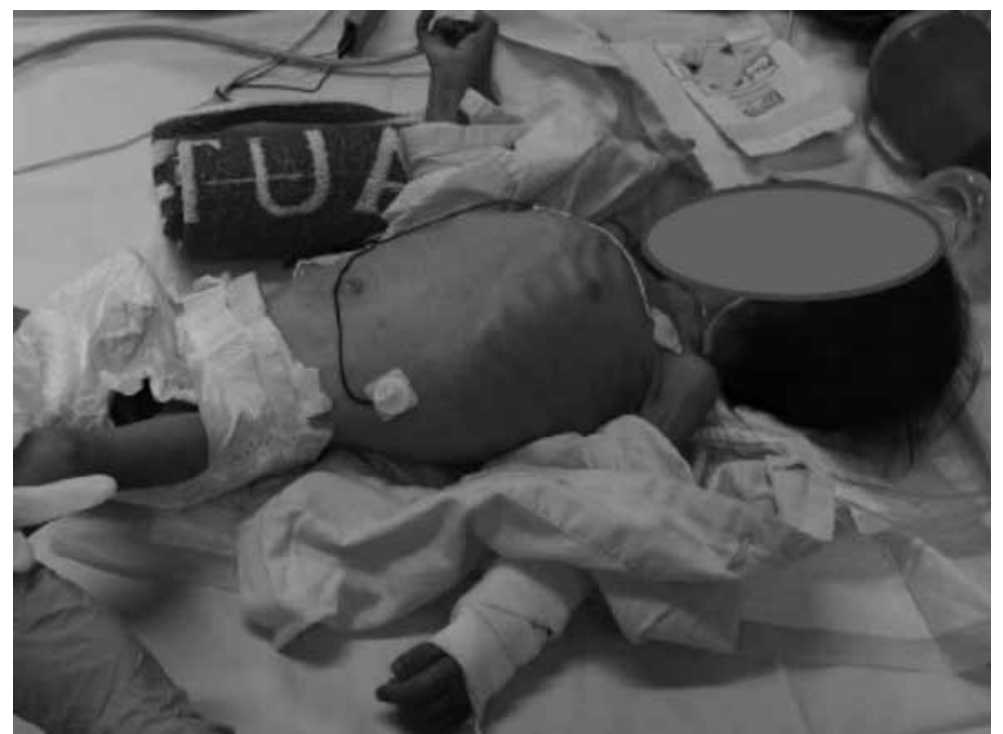

FIG I. Cachexic girl with pneumonitis

2. Complement C3 and C4 levels for congenital complement deficits

3. Immunoglobulin A for immunoglobinopathy

4. H7N9, coronavirus, mycoplasma, and chlamydia serology for atypical pneumonia

5. HIV testing

After performing various investigations (Table), the child tested positive for HIV infection, confirming that she had acquired immunodeficiency syndrome (AIDS). Computed tomographic scan of thorax showed pneumonitis (Fig 3). Treatment for Pneumocystis jiroveci pneumonia and cytomegalovirus infection was commenced. Following stabilisation, the child was referred to a paediatric infectious disease specialist for continuation of care. Highly active antiretroviral therapy was started when opportunistic infections were under control. The child was last seen in June 2014; she was asymptomatic and had been thriving well.

The parents refused HIV testing but reported that the child had received blood products after onset of illness while in the Mainland hospital. Both were subsequently confirmed HIV positive. Nevertheless, HIV was most likely to be vertically (mother to child) transmitted. $^{2-5}$ In many areas of the world, HIV/AIDS has become a chronic rather than an acutely fatal

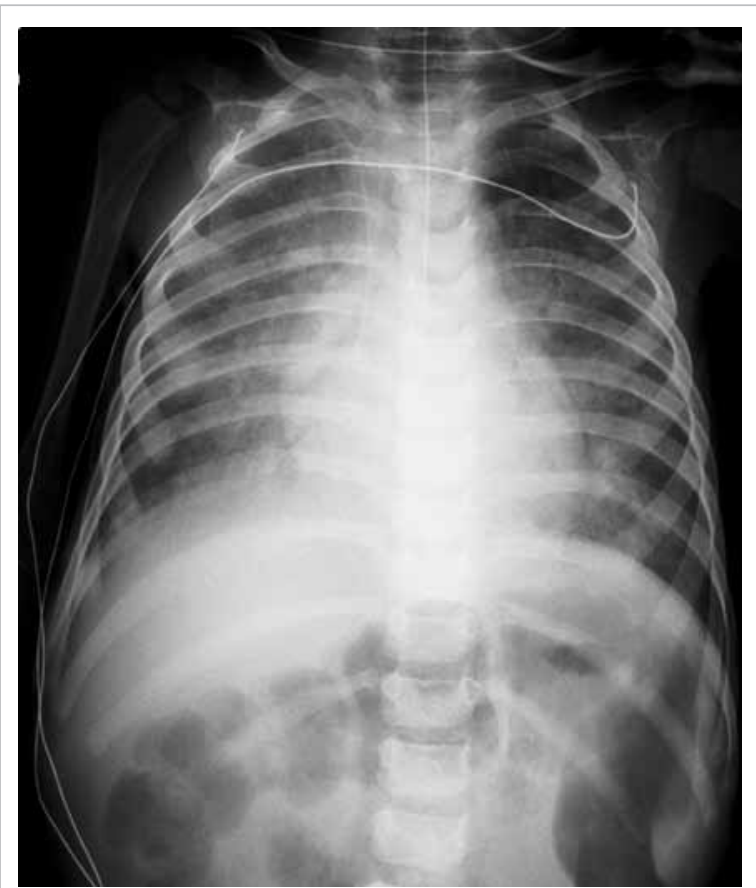

FIG 2. Chest X-ray showing fulminant pneumonitis 
TABLE. Some relevant investigations performed in the patient

\begin{tabular}{lll}
\hline Test & Results & Remarks \\
\hline Anti-HIV antibody & Strongly positive & Unreliable during infancy \\
Western blot & Positive & Sensitive and specific test for HIV antibodies \\
Bronchoalveolar lavage cytology & $\begin{array}{l}\text { PCP seen, detection of early antigen fluorescent foci } \\
\text { (DEAFF) for CMV positive }\end{array}$ & Pneumocystis and CMV pneumonias are common \\
pD4/CD8 & 0.14 & Normal >1 \\
IgG, IgA, IgM & Elevated & Elevated in most patients with HIV/AIDS \\
Serial CMV pp65 & Positive & A rapid CMV pp65 antigenemia assay \\
Beta-D-1,3-glucan & Strongly positive & Test for fungal infection (positive also in PCP infection) \\
Mantoux test & Non-reactive & Test for mycobacterial infection \\
Serial gastric aspirates & No acid-fast bacillus & Culture for mycobacteria
\end{tabular}

Abbreviations: AIDS = acquired immunodeficiency syndrome; CMV = cytomegalovirus; HIV = human immunodeficiency virus;

Ig $=$ immunoglobulin; PCP = Pneumocystis jiroveci pneumonia

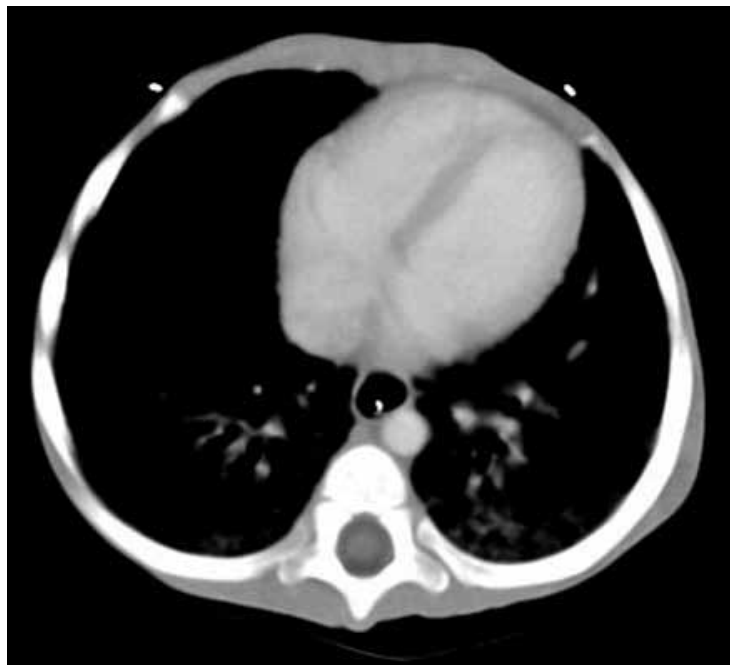

FIG 3. Computed tomography thorax with contrast showing pneumonitis with diffuse ground-glass opacities and interseptal thickening, but no lung consolidation or hilar lymphadenopathy

disease. ${ }^{3}$ Half of the infants born with HIV die before 2 years of age without treatment..$^{2-5}$

Pneumonitis is usually caused by viruses or atypical pathogens. Since the atypical pneumonitis epidemic of coronavirus in 2003, Hong Kong and the rest of the world have heightened surveillance for outbreaks of atypical pneumonitis with novel pathogens such as avian or swine influenza, or coronavirus. ${ }^{1}$ In the cosmopolitan city of Hong Kong, paediatric HIV remains a relatively rare diagnosis. Nevertheless, due to the busy trafficking between Hong Kong and Mainland China, paediatricians in Hong Kong must be vigilant of such possibility in Hong Kong children of Mainland parents. Despite the misleading history by the parents about negative screening for HIV, paediatricians at the paediatric ICU were prompt to arrive at the definitive diagnosis by requesting HIV testing and considering HIV infection as a possibility to explain the combination of pneumonitis and extreme failure to thrive in this child.

This case is interesting and highlights the importance of excluding HIV infection in a child with dual symptoms of recurrent infections and failure to thrive, even when the mother had tested negative for HIV antibodies initially; the test may have been performed in a window period during pregnancy.

Differential diagnoses for failure to thrive include child abuse and neglect, cystic fibrosis (rare in Hong Kong), gastroesophageal reflux, growth failure, growth hormone deficiency, and HIV infection. ${ }^{6,7}$ The history and physical examination should guide any laboratory or ancillary testing. Most infants and children with growth failure related to environmental factors need very limited laboratory screenings. This child presented with recurrent infections and candidiasis. Approach to recurrent infections resulting in failure to thrive may include HIV testing, sweat test for cystic fibrosis (if history is relevant), metabolic and endocrinology screening, tuberculosis testing, and stool studies.

Basing on disease onset, this is most likely a case of vertical transmission of HIV. In infants, the onset of AIDS symptoms can take a few months; in contrast, it can be many years before adults develop symptoms of HIV. Thus, repeated HIV testing is very important to initiate timely treatment in the parents.

\footnotetext{
${ }^{1}$ KL Hon *, MD, FCCM

${ }^{1}$ TF Leung, FRCPCH, FHKAM (Paediatrics)

${ }^{2}$ YS Yau, MRCP (UK), FHKAM (Paediatrics)
}

Department of Paediatrics, The Chinese University of Hong Kong, Prince of Wales Hospital, Shatin, Hong Kong 
2 Department of Paediatrics, Queen Elizabeth Hospital, Jordan, Hong Kong

* Corresponding author: ehon@cuhk.edu.hk

\section{References}

1. Hon KL. Severe respiratory syndromes: travel history matters. Travel Med Infect Dis 2013;11:285-7.

2. Sepkowitz KA. AIDS-the first 20 years. N Engl J Med 2001;344:1764-72.

3. Knoll B, Lassmann B, Temesgen Z. Current status of HIV infection: a review for non-HIV-treating physicians. Int I Dermatol 2007;46:1219-28.

4. Coutsoudis A, Kwaan L, Thomson M. Prevention of vertical transmission of HIV-1 in resource-limited settings. Expert Rev Anti Infect Ther 2010;8:1163-75.

5. Thorne C, Newell ML. HIV. Semin Fetal Neonatal Med 2007;12:174-81.

6. Nangia S, Tiwari S. Failure to thrive. Indian J Pediatr 2013;80:585-9.

7. Hendaus $\mathrm{M}, \mathrm{Al}-$ Hammadi A. Failure to thrive in infants (review). Georgian Med News 2013;(214):48-54. 